\title{
What technique is suitable for laparoscopic suprapancreatic lymph node dissection?
}

\author{
Seigo Kitano \\ Department of Surgery I, Oita University Faculty of Medicine, 1-1 Idaigaoka, Yufu, Oita 879-5593, Japan
}

Recently, laparoscopy-assisted distal gastrectomy (LADG) with lymph node dissection has become popular as a procedure for gastric cancer in Japan and Korea. According to a national survey conducted by the Japan Society of Endoscopic Surgery (JSES), more than 15000 patients with gastric cancer underwent LADG from 1991 to 2007 in Japan [1, 2]. The present indication for LADG is early gastric cancer, with the risk of lymph node metastasis, which is not suitable for endoscopic submucosal dissection (ESD). In Asian countries, D2 or D2 modified lymph node dissection (D1+No.7, 8a, 9, $11 p$ ) is usually performed during LADG. As the number of patients undergoing LADG has been increasing, evaluation of the surgical outcomes of this operation has begun. There are four randomized controlled trials (RCTs) and several retrospective studies of the outcomes of LADG. The meta-analysis by Memon et al. shows that the number of dissected lymph nodes in LADG is lower than that in open gastrectomy [3]. Indeed, Miura et al. show in a retrospective study that the number of dissected suprapancreatic lymph nodes (No. 9 and 11p lymph nodes) is smaller in LADG than in open gastrectomy [4]. Therefore, it is necessary to establish safe techniques of suprapancreatic lymph node dissection in LADG with D2 or D2 modified lymph node dissection. The new technique of a left-sided approach to suprapancreatic lymph node dissection introduced in the article by Fukunaga et al. in this issue [5] may be useful for complete suprapancreatic lymph node dissection.

There are technical disadvantages to laparoscopic surgery: surgical procedures are conducted under limited 2-D vision, with limited movement, by long forceps and no sensation of touch. These characteristics make laparoscopic lymph node dissection difficult. In laparoscopic lymph node dissections during LADG, the suprapancreatic lymph node dissection may be the most

Offprint requests to: S. Kitano difficult. In conventional LADG, techniques of taping vessels and pushing the pancreas downward after the duodenal transection are often used for complete dissection of lymph nodes around the common and proper hepatic arteries as well as the splenic artery [6]. In laparoscopic suprapancreatic lymph node dissection, better methods are required for improving the operative field and handling the lymph node dissection. In their article, Fukunaga et al. [5] emphasize that not transecting the duodenum inside the abdominal cavity is useful for obtaining a clear view of the operative field during LADG.

In suprapancreatic lymph node dissection, creating a better operative field is fundamental for removing the lymph nodes completely and safely. The new technique by Fukunaga et al. [5] is described as an expansion of the hepatoduodenal ligament and the left gastropancreatic fold without transection of the duodenum, which achieves an improved field of view. Fukunaga et al. [5] show that for 12a lymph node dissection, the hepatoduodenal ligament can be expanded easily by pulling strongly on the pyloric region of the stomach in the left caudal direction owing to omission of duodenal transection. Also, the authors demonstrate that for No. 9 and $11 \mathrm{p}$ lymph node dissection, the gastropancreatic fold is easily maneuvered ventrally through the opened lesser sac after the dissection of lymph nodes on the left side of the left gastric vessels (left-sided approach). Additionally, for No. 8a lymph node dissection, stretching of the gastropancreatic fold causes expansion of the peritoneum covering the No. 8a lymph node, and the leftsided approach without duodenal transection suggested by the authors provides a dry operative field without bleeding and lymph fluid leakage. To create an operative field for laparoscopic techniques in the suprapancreatic area, retracting the stomach to enable expansion of the hepatoduodenal ligament and stretching the gastropancreatic fold may be more useful than taping the vessels or pushing the pancreas downward. 
Ryu et al. [7] evaluated surgical outcomes of 347 LADGs and reported that both the extent of lymph node dissection and surgical inexperience are risk factors for operative complications associated with LADG. Dissection of suprapancreatic lymph nodes is required during extended lymph node dissection of D2 or the D2 modified technique. It is possible that suprapancreatic lymph node dissection causes an increase in the incidence of operative complications, including pancreatic fistula in particular. Fukunaga et al. [5] analyzed the surgical outcomes of 391 patients who underwent LADG with the new techniques of laparoscopic lymph node dissection. The authors showed that the number of lymph nodes dissected with the new technique was comparable to the number with open gastrectomy [3], and that the duration of the operation was shorter than that of conventional LADG. The amount of blood loss was smaller and the incidence of complications including pancreatic fistula was lower than that of open surgery. These improved results may support the feasibility of the new laparoscopic procedures for suprapancreatic lymph node dissection.

Obesity is believed to be a serious risk in the successful completion of LADG [8]. In LADG for obese patients, suprapancreatic lymph node dissection is sometimes technically difficult because the borderline between the upper edge of the pancreas and the fat tissue containing the lymph nodes is not clear and because bleeding and leakage of lymph occurs easily from the cut edge of thick fat tissue. The study described by Fukunaga et al. [5] included 8 patients with BMI over 30 and as high as 35.6. The authors showed that all obese patients safely underwent LADG via the leftsided approach to suprapancreatic lymph node dissection without duodenal transection, and speculated that the new technique may be more suitable for obese patients than the conventional LADG because a clear operative field is easily obtained by retraction of the left gastropancreatic fold from the surrounding tissues. In the near future, this issue should be clarified by analyzing outcomes of a larger number of obese patients who have undergone this new technique.

For surgical staff there is a well-defined learning curve recognized during acquisition of LADG techniques. It has been reported by Kim et al. [9] and Fujiwara et al. [10] that surgeons need to experience more than 50 cases to achieve a plateau in this learning curve. Fukunaga et al. [5] stated that it was easier for both surgeons and assistants to acquire proficiency in the left-sided techniques than with the conventional procedure: requiring experience of approximately 10 cases to establish proficiency for the former. The reason for this shorter learning period is unclear. It may be that standardization or the mode of education in this new technique is well organized. At the same time, the period needed for acquisition of laparoscopic procedures may depend on the operative approach.

In conclusion, the new method of a left-sided approach to suprapancreatic lymph node dissection without duodenal transection seems to be convenient and useful in LADG. For popularization of this new technique, an oncologic evaluation must be undertaken and technical feasibility confirmed by a number of treatment settings in the near future.

\section{References}

1. Kitano S, Iso Y, Moriyama M, Sugimachi K. Laparoscopy-assisted Billroth I gastrectomy. Surg Laparosc Endosc 1994;4:146-8.

2. Japan Society for Endoscopic Surgery. Nationwide survey on endoscopic surgery in Japan. J Jpn Soc Endosc Surg 2008;13:499_ 604 (in Japanese).

3. Memon MA, Khan S, Yunus RM, Barr R, Memon B. Metaanalysis of laparoscopic and open distal gastrectomy for gastric carcinoma. Surg Endosc 2008;22:1781-9.

4. Miura S, Kodera Y, Fujiwara M, Ito S, Mochizuki Y, Yamamura $\mathrm{Y}$, et al. Laparoscopy-assisted distal gastrectomy with systemic lymph node dissection: a critical reappraisal from the viewpoint of lymph node retrieval. J Am Coll Surg 2004;198:93-8.

5. Fukunaga T, Hiki N, Tokunaga $M$, Nohara $\mathrm{K}$, Akashi $\mathrm{Y}$, Katayama $\mathrm{H}$, et al. Left-sided approach for suprapancreatic lymph node dissection in laparoscopy-assisted distal gastrectomy without duodenal transection. Gastric Cancer 2009;12:106-12.

6. Tanimura S, Higashino M, Fukunaga Y, Takemura M, Tanaka Y, Fujiwara Y, et al. Laparoscopic gastrectomy for gastric cancer: experience with more than 600 cases. Surg Endosc 2008; 22:1161-4.

7. Ryu KW, Kim YW, Lee JH, Nam BH, Kook MC, Choi IJ, et al. Surgical complications and the risk factors of laparoscopy-assisted distal gastrectomy in early gastric cancer. Ann Surg Oncol 2008;15:1625-31.

8. Noshiro H, Shimizu S, Nagai E, Ohuchida K, Tanaka M. Laparoscopy-assisted distal gastrectomy for early gastric cancer. Is it beneficial for patients of heavier weight? Ann Surg 2003; 238:680-5.

9. Kim MC, Jung GJ, Kim HH. Learning curve of laparoscopyassisted distal gastrectomy with systemic lymphadenectomy for early gastric cancer. World J Gastroenterol 2005;11:7508-11.

10. Fujiwara M, Kodera Y, Miura S, Kanyama Y, Yokoyama H, Ohashi N, et al. Laparoscopy-assisted distal gastrectomy with systemic lymph node dissection: a phase II study following the learning curve. J Surg Oncol 2005;91:26-32. 